\title{
El material epigráfico de Tell El-Ghaba (Norte de Sinaí, Egipto)
}

\author{
María Violeta Pereyra*
}

\begin{abstract}
PEREYRA, M.V. El material epigráfico de Tell El-Ghaba (Norte de Sinaí, Egipto). Revista do Musen de Arqueologia e Etnologia, São Paulo, 19: 257-271, 2009.
\end{abstract}

Resumo: $\mathrm{O}$ objetivo deste estudo é interpretar o material epigráfico descoberto em Tell El-Ghaba no decorrer das escavações realizadas entre 1995 e 2000 pela Missão Arqueológica Argentina. A partir da catalogação dos achados, a tradução e interpretação das inscrições e a decodificação da iconografia, os motivos decorativos se compararam com outros similares dispostos sobre objetos com epigrafia procedentes de sítios contemporâneos e, finalmente, foram estudados sobre fundamentos bibliográficos. Como resultado, pode-se observar que: 1- a natureza dos amuletos analisados em muitos casos é pictográfica; 2-os textos registrados sobre os amuletos se vinculam principalmente com a teologia de Amon; 3- a maioria dos achados pertence à área I, de caráter industrial e residencial.

Palavras-chave: Tell el-Ghaba - Epigrafia - Amuleto - Escaravelho - Olho de Hórus - Criptografia.

\section{Introducción}

E n la antigüedad, el sitio arqueológico excavado en la moderna Tell el-Ghaba (30॰ $58^{\prime}$ de latitud norte y $32^{\circ} 25^{\prime}$ de longitud oeste) era un establecimiento estatal erigido en la frontera oriental de Egipto luego de su expansión hacia el Levante en Ėpoca Baja. ${ }^{1} \mathrm{La}$ información que provee la cerámica de las áreas excavadas (Fuscaldo et al. 2002) sugiere que la

(*) Universidad de Buenos Aires - Consejo Nacional de Investigaciones Científicas y Técnicas.

violetapereyra@yahoo.com.ar

(1) De acuerdo a las dimensiones y características

arquitectónicas de algunos edificios identificados en el sitio y las vasijas de almacenamiento descubiertas en él (Crivelli Montero, Cohen y Chauvin 2002: 836-839; Crivelli Montero 2006: 17-31). historia del sitio se inserta en el comienzo del Período Saíta (siglos VII-VI a.C.) y es probable que las estructuras descubiertas deban interpretarse a partir de la función defensiva que tuvo, pero también teniendo en cuenta la red de tráfico que los soberanos saítas extendieron. Adyacente a la ruta costera hacia el Levante, la cerámica importada de Tell el-Ghaba indica fuertes conexiones con el Mediterráneo oriental ${ }^{2}$ y con una vía de circulación que seguía el brazo Pelusíaco del Nilo, lo que refuerza la afirmación anterior.

La economía del área debió basarse en la agricultura y la evidencia de especialización

(2) Sin variaciones notables entre los cuatro niveles de ocupación del sitio, fuera de su incremento en el más reciente. 
artesanal se habría limitado a la producción cerámica y textil. El grado de dependencia de Tell el-Ghaba respecto de la administración central es una cuestión abierta, aunque es muy probable que, por lo menos hasta el Período del Bronce Reciente, la economía del norte de Sinaí mantuviera ese carácter. Los bienes requeridos por los egipcios asentados en la zona pudieron haber sido producidos localmente, mientras que las fortalezas y centros administrativos serían provistos por el sistema distributivo del estado egipcio. ${ }^{3}$

La localización del sitio es de importancia sustantiva desde el punto de vista estratégico, a la vez que se trata de un área cuya densidad de población es baja. Ubicado en la vecindad del Camino real ${ }^{4}$ que comunicaba el Valle del Nilo con Palestina, la vida del asentamiento de época saíta se extendió por más de un centenar de años, en los que sirvió en forma sucesiva como lugar de frecuentación y de ocupación permanente, de acuerdo a las condiciones políticas de cada momento. Los materiales de Tell el-Ghaba que nos ocupan se fecharon en el período en que funcionó como centro administrativo e industrial, ${ }^{5}$ hasta su destrucción a comienzos de la época persa (siglo VI). No obstante, las evidencias de la periferia indican que fue ocupada antes y después del período saíta (Pereyra y Zingarelli 2002: 861).

Las áreas investigadas por la Misión Argentina fueron ocho (véase Fig. 1), procediendo la totalidad de los materiales que estudiamos de las áreas I y II.

\section{La colección, consideraciones generales}

Las excavaciones realizadas por la misión Arqueológica Argentina en Tell el-Ghaba entre

(3) En el sitio hay indicios de actividades comerciales y administrativas (pesas de piedra pulida, discos de cerámica usados para cálculos), además de industriales (restos de hornos de diversos tipos y escoria).

(4) El "Camino de Horus" en egipcio. Así denominado desde el Reino Medio (Pereyra y Zingarelli 2002: 859; Zingarelli 2005: 171-184).

(5) La fase IV Norte según la datación cerámica asociada, que comprendería los reinados de Psamético a Apries (Fuscaldo 2007: 31)
1995 y 1999 permitieron recuperar un conjunto de materiales con epigrafía que oportunamente fueron estudiados por Silvana Fantechi y la autora. ${ }^{6}$

La colección de hallazgos especiales con epigrafía del sitio está compuesta en su mayor parte por amuletos-sello de dimensiones reducidas, ${ }^{7}$ con dos excepciones. El conjunto se compone de doce escarabajos, ${ }^{8}$ dos escaraboides, ${ }^{9}$ tres plaquetas, ${ }^{10}$ un udyat ${ }^{11} \mathrm{y}$ un amuleto-sello zoomorfo. ${ }^{12}$

Los otros dos objetos que completan los materiales con epigrafía de Tell el-Ghaba corresponden a la sección de un torso de estatuilla real (?) de faenza ${ }^{13}$ y un sillar con un relieve fragmentario que preserva un diseño lineal figurativo. ${ }^{14}$ Ambos fueron publicados en el catálogo del sitio (Fuscaldo 2007) y carecen del sentido protector del conjunto de materiales epigráficos aquí tratados. Esa diferencia justificó su omisión en este estudio.

La documentación de la evidencia iconográfica e inscripta que conservaron los objetos estudiados se realizó en el curso de las sucesivas campañas de trabajo en Egipto y su registro fue cualitativamente dispar. ${ }^{15}$ No obstante, en la mayoría de los casos la documentación que sirvió de base al estudio de los materiales

(6) El documento Catalogue of Scarabs, Scaraboids, Button Seals and Plaquets with Ephigraphy (inédito) fue preparado a partir de la documentación llevada a cabo por la Misión Arqueológica Argentina y concluido en 2004. Algunos fueron recientemente publicados (Fuscaldo 2007: vol. 2) (7) La máxima dimensión registrada alcanza los $3,15 \mathrm{~cm}$. (8) Números de inventario: F0015, F0019, F0083, F0166, F0274, F0434, F0622, F0629, F0636, F0640, F0650 y F0655.

(9) Números de inventario: F0092 y F0492.

(10) Números de inventario: F0235 y F0491, F118.

(11) Número de inventario: F1000.

(12) Número de inventario: F0072.

(13) Número de inventario F0280. El fragmento, de 2,41 x 1,6 × 1,22 (color: 10 YR 8/4 very pale brown), conserva restos de una inscripción casi ilegible aA-ib-ra (?).

(14) Número de inventario F0773. Se encontró en la base de un muro de adobe (L1138) durante la excavación del edificio D. El bloque es de caliza, está decorado en bajo relieve y las líneas preservadas permiten reconocer la silueta de una figura humana sobre una barca que se desplaza en un medio nilótico (Fuscaldo 2007: Fig. 37, No. 1). (15) Diversas circunstancias condicionaron la disponibilidad de documentación fotográfica, faltante por completo en algunos casos, mientras que en otros los registros escritos son incompletos y los gráficos sólo esquemáticos. 


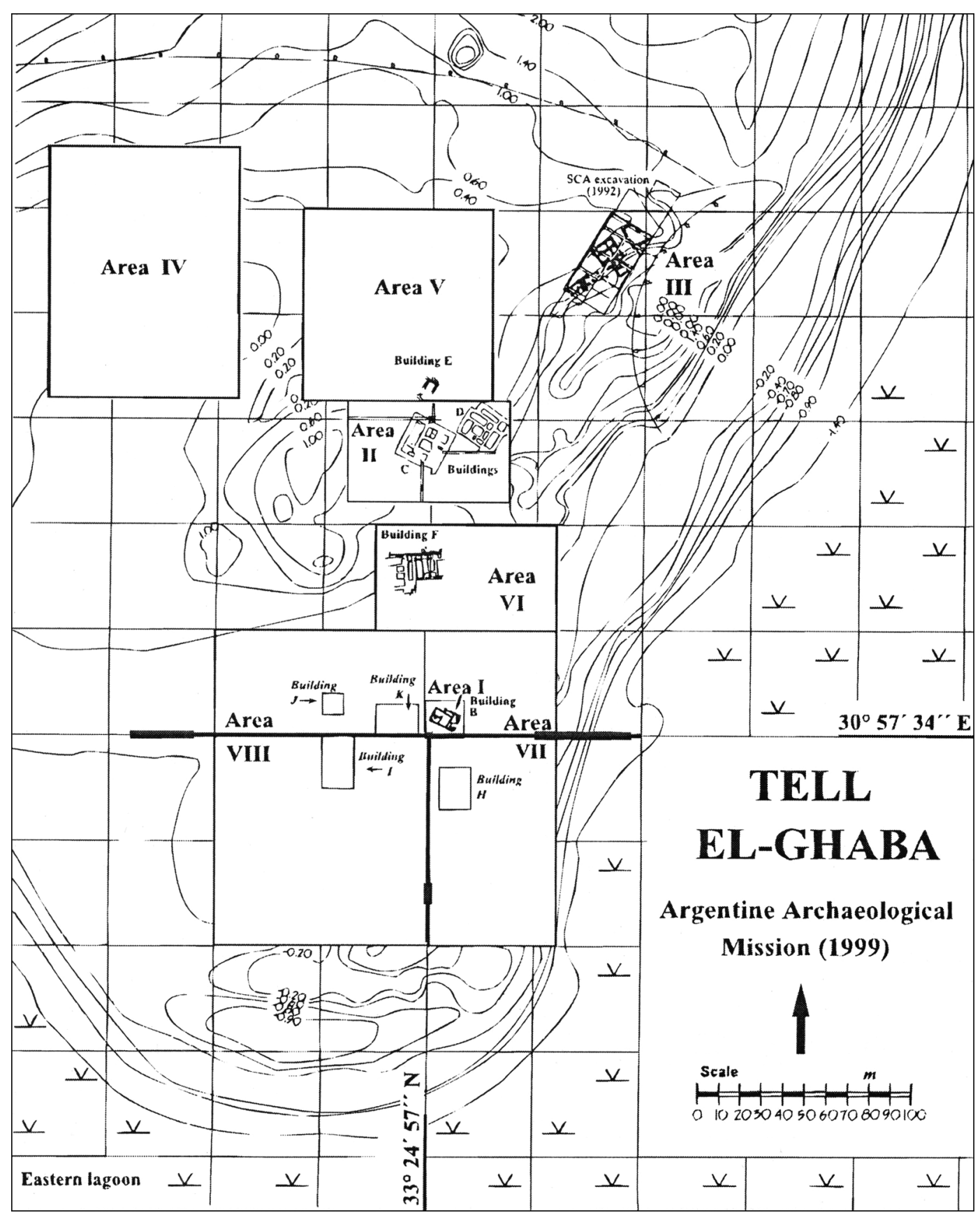

Fig. 1. Áreas relevadas y excavadas en Tell el-Ghaba (Fuscaldo 2007: Plan 1).

epigráficos está representada por registros escritos, gráficos (dibujo en escala 1:1) y fotográficos de cada objeto (véase Fig. 2).

De manera excepcional, contamos además con las improntas de algunos escarabajos y escaraboides realizadas en base a moldes tomados en la campaña de 1998 (véase Fig. 3). Su disponibilidad facilitó notablemente el reconocimiento de los diseños que decoraban esos ejemplares. 

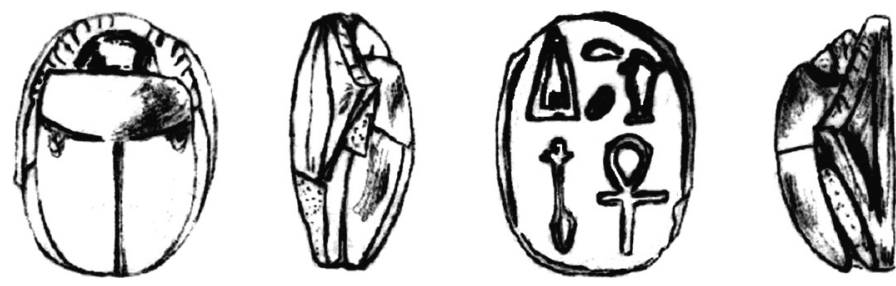

Fig. 2. Registro en dibujo del escarabajo F0650.
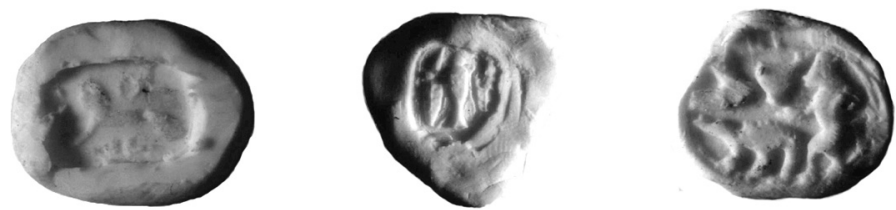

Fig. 3. Improntas de decoración basal (F0019, F0166 y F0015).

las plaquetas es vertical, coincidente en ambos casos con el eje mayor de los objetos. Lo mismo ocurre con el udyat y el amuleto zoomorfo.

Para su tratamiento, agrupamos los materiales: en primer lugar de acuerdo al tipo de objeto al que pueden adscribirse y segundo por el área de su descubrimiento en Tell elGhaba. Cabe mencionar que si bien de las ocho áreas definidas en el sitio las investigadas fueron siete (Fuscaldo 2007: Plan 1), hasta la fecha sólo en dos de ellas se identificaron materiales

Los materiales con que fueron elaborados los amuletos de Tell el-Ghaba se limitan a piedras (esteatita y diorita) y faenza. La mayor parte de ellos es de faenza y fueron manufacturados con moldes y decoración incisa, en tanto que los de piedra se decoraron con motivos iconográficos e inscripciones talladas en bajo relieve.

En la decoración de este conjunto de amuletos, escarabajos, escaraboides y plaquetas fueron utilizados signos figurativos y jeroglíficos que forman composiciones simbólicas o inscripciones criptográficas. Entre ellas podemos distinguir aquellas decoradas con signos puramente figurativos - divinidades o elementos icónicos - y las que utilizaron signos del sistema jeroglífico de escritura, aunque en muchos casos con valores alterados.

A pesar de las diferencias que individualmente muestran las diversas categorías de objetos, los escarabajos, escaraboides y plaquetas con regularidad tienen perforaciones que permiten inferir un uso potencial como anillos de sello o cuentas para enhebrar en un collar. ${ }^{16}$ En el caso de los escarabajos y escaraboides la perforación es longitudinal, en tanto que la de

(16) En la campaña de 1995 se encontraron en el área I (L0089) 156 piezas pertenecientes a un collar de 152 caracolas y 4 udyats (F0159) (Rosso, Fantechi y Arbolave 2002: 877). con epigrafía: las áreas I y II. Las características que reviste la ocupación de una y otra área en el período asociado a los hallazgos son diferentes, la primera residencial e industrial y la segunda de carácter público.

Del área I proceden ocho escarabajos (F0015, F0019, F0083, F0622, F0629, F0636, F0650 y F0655), dos escaraboides (F0092 y F0492), el amuleto-sello zoomorfo (F0072), el udyat (F1000) y dos plaquetas (F0235, F0491), mientras que los cuatro escarabajos restantes (F0166, F0274, F0434, F0640) y una plaqueta (F0118) fueron descubiertos en el área II. También el bloque decorado en relieve (F0773) y el fragmento de estatuilla con inscripción (F0280), aquí omitidos, proceden de esta área.

\section{Dos amuletos de Tell el-Ghaba con epigrafía (F0072 y F1000)}

El único ejemplar de un amuleto-sello con epigrafía que difiere morfológica y funcionalmente del resto es evocativo de la diosa Heket. En época faraónica su iconografía adoptó la rana del Nilo para representarla directamente por su figuración zoomorfa o como una mujer con cabeza de rana o que lleva una rana como tocado. Asociada con el descenso de la inundación y las aguas primordiales de la precreación, la diosa Heket asumió un rol eminentemente creativo en la mitología egipcia, 
como fuente de vida y de renovación. ${ }^{17}$ Como contraparte de Khnum, que insuflaba vida al individuo modelado por el dios alfarero, se convirtió en protectora de los nacimientos y por extensión del renacimiento y de la fertilidad humana. ${ }^{18}$ En consecuencia, las representaciones de la diosa Heket fueron empleadas como talismanes propiciatorios y protectores del parto por las mujeres egipcias, pero también como amuleto funerario que aseguraba el renacimiento de los difuntos.

Los tres amuletos de Heket de Tell el Ghaba proceden de las áreas I y II, pero ninguno se encontró asociado a un contexto definido. Dos de ellos son anepigráficos (F0063 y F0278), en tanto que el que nos ocupa aquí (F0072) tiene la forma de una rana apoyada en una plataforma redondeada en cuya base se conserva, aunque poco legible, una inscripción jeroglífica.

El amuleto de Heket (FO072) procede del área I y el locus en que se encontró (L0008) no está asociado a ninguna estructura. Es un locus carbonoso que comparte con el que lo cubría (L0001) su carácter de estrato de destrucción. ${ }^{19}$ Fue tallado en esteatita (color: 7.5YR 6/4 light brown) y su dimensiones (en $\mathrm{cm}$ ) son $0.8 \times 0,65$ y 0,5 (véase Fig. 4). Este ejemplar de Tell elGhaba es semejante a otro del área menfita (Petrie 1925: Pl. I, P2).
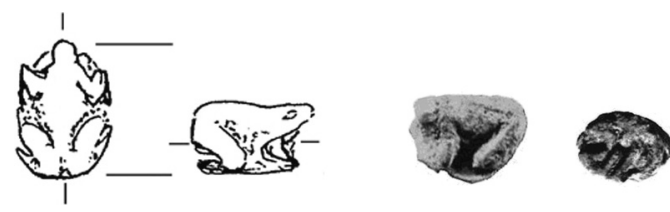

Fig. 4. Amuleto F0072.

(17) Por eso la figura de la rana fue usada como signo jeroglífico para representar la idea de renovación en general, como en la expresión $w h{ }^{\text {` }} n h$ (véase signo I7 de la lista de Gardiner).

(18) La Recitación 539 (1312 c-d) de los Textos de las Pirámides hace referencia a Heket, identificada con las partes del rey entorpecidas en su ascenso al cielo; el Papiro Westcar, por su parte, la menciona en el episodio del nacimiento de los tres primeros reyes de la dinastía $V$; y la teogamia representada en el templo de Hatshepsut en Deir el Bahari, evoca los poderes de la diosa. Estos tres ejemplos muestran la antigüedad del culto y su continuidad a lo largo de la historia faraónica hasta el período saíta.
Otro tipo de amuleto del que se encontraron más de cincuenta ejemplares en Tell el-Ghaba es el $u d y a t$, considerado en el antiguo Egipto como un protector muy poderoso que se vinculó a las ideas de restitución, regeneración y abundancia. De acuerdo a los textos mitológicos, en época tardía los udyats representaban los ojos del cielo: el derecho al sol y el izquierdo a la luna (Rosso, Fantechi y Arbolave 2002: 877). El Ojo de Ra se convirtió en símbolo de lo inalterable y eterno, en tanto que el de Horus, asociado a la luna, evocaba el poder de renovación representado en la mitología como el conflicto entre Horus y Seth, y en el ritual como el arquetipo de la ofrenda filial. ${ }^{20}$

La colección de Tell el-Ghaba es variada y la mayoría procede del área I. Algunas piezas corresponden al ojo derecho, el Ojo de Ra, y otras al izquierdo, el Ojo de Horus (Rosso, Fantechi y Arbolave 2002: fig. 4), en tanto que también hay ejemplos de udyats cuádruples, como por ejemplo F0007A (Fuscaldo 2007: Plate 20, No 426; Rosso, Fantechi y Arbolave 2002: fig. 5).

Una gran cantidad de variantes morfológicas de udyats manufacturados en faenza y piedra está ampliamente representada en los ejemplares del sitio (Fuscaldo 2007: Plate 19-23, Nos.425. 429, Plate 24-27, Nos.431-435 y Fig. 40: 37 , No.438). Hasta la fecha sólo se identificó un udyat (F1000) con una inscripción jeroglífica registrada en su reverso (Fuscaldo 2007: Plate 6, No. 51) que fue concebido en forma de cuenta de collar, como la casi totalidad del conjunto (Fuscaldo 2007: Plate 6 y 7, Nos. 50 y 51). ${ }^{21} \mathrm{El}$ otro ejemplar epigráfico es cuadrangular y lo analizamos más abajo, integrado al grupo de las plaquetas (F118).

(19) En el L0008 se encontraron además 16 udyats de faenza. (20) En el Reino Nuevo, en el capítulo 42 del Libro de los Muertos se registra ese conflicto entre la luz, evocada por los Ojos del Cielo, y la oscuridad del inframundo, identificándose el difunto con el ojo.

(21) Un udyat de piedra con talla sin terminar que procede del área II (L1043) (Fuscaldo 2007: Plate 2, No. 27 -F0251) y una serie de $u$ dyats de variada factura se publicaron en el catálogo del sitio (Fuscaldo 2007: Plate 11-12, Nos 148 . F0013A y No. 149 -F0165A). 
El udyat F1000 procede de L0271,22 un locus asociado a un piso del edificio B (L0017), nivel IV del área I y fue descubierto junto a otros hallazgos especiales, entre los que se destacan un amuleto con la figura del dios Bes (F009) y una cuenta de oro (F008) de excelente factura (Fuscaldo 2007: Plates 8 y 9 Nos. 52 y 53). Es el amuleto con epigrafía de mayores dimensiones del sitio: $3,15 \mathrm{~cm} \times 2,10 \mathrm{~cm} \times 0,4$ $0,7 \mathrm{~cm}$; es de faenza color blanco y su decoración incisa se destacó con líneas negras (véase Fig. 5).
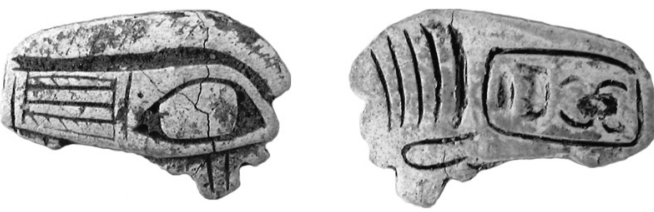

Fig. 5. Udyat F1000.

La forma de este udyat corresponde a la del Ojo de Ra y sus dos caras están decoradas. En el anverso, levemente convexo, están incisos el globo ocular y la ceja del ojo de Horus, además de un diseño lineal en el interior de un rectángulo (un serekh (?) estilizado). El reverso es plano y lleva una inscripción jeroglífica incisa en el interior de una cartela que reza $m n-h p r-r^{\complement}$, junto a un patrón ornamental de cinco líneas verticales incisas en forma paralela y ligeramente curvadas hacia el borde posterior. Por su inscripción, es posible vincularlo a los escarabajos del tipo "menkheperra", que están bien atestiguados en las dinastías XXV y XXVI (Jaeger 1982: 246-253) y de los que se encontraron varios ejemplares en Tell elGhaba. Aún cuando la expresión "menkheperra" se empleó para representar en forma criptográfica el trigrama de Amón, cuyo nombre significaba "El Oculto", su inclusión en este caso en una cartela y la presencia de los halcones que la enmarcan también hacen posible su atribución a algún soberano histórico que adoptó el nombre de Thutmosis III. ${ }^{23}$

(22) Asociado al L0017 por error en Fuscaldo 2007: Plate 6. (23) El rey kushita Piankhi y el saíta Necao (Jaeger 1982: 252).
Además de la inscripción que porta, F1000 se distingue del resto de la serie de udyats descubiertos en Tell el-Ghaba por la concepción de su diseño y porque su factura y terminación son muy cuidadas, a pesar de la simplicidad de su forma y de lo esquemático de su decoración.

\section{Las plaquetas de Tell el-Ghaba (F0491, F0235 y F0118)}

Tres son las piezas descubiertas en Tell elGhaba que podemos agrupar en la categoría de plaquetas. Dos de ellas son de esteatita y fueron decoradas con figuras grabadas con líneas de contorno, mientras que la tercera es de diorita y su decoración fue hecha con talla en bajo relieve.

Dos plaquetas proceden del área I: F0491 y F0235, y una del área II: F0118.

La primera de las plaquetas del área I, F0491 (véase Fig. 6) se encontró en un nivel de destrucción (L0001). Es de faenza (color 7.5YR $5 / 3$ brown) y mide $1,5 \mathrm{~cm} \times 1,1 \mathrm{~cm} \mathrm{x} \mathrm{0,6} \mathrm{cm.}$ Presenta sus dos caras decoradas con incisiones profundas y tanto el anverso como el reverso tienen composiciones en las que se emplearon signos figurativos y jeroglíficos (Fuscaldo 2007: Plate 29, No. 441). La inscripción del anverso es criptográfica ${ }^{24}$ y debe leerse imn, "Amón", atribuyendo en su decodificación los respectivos valores $i$ y $m n$ a la pluma de Maat y el dios Montu representados allí sobre una línea de base. La inscripción del reverso, por su parte, fue escrita de acuerdo a los códigos usuales de la escritura jeroglífica y dice: $m n$ imn "Amón permanece" (lit. "Permanente es Amón”).

La segunda plaqueta del área I (F0235) es de diorita y se encontró en el sedimento contenido en una vasija levantina perteneciente al depósito de fundación del edificio B (L0211) (Fuscaldo 2007: Plate 4 No. 10). De color oscuro (N4 dark gray) y aspecto lustroso, es similar a la anterior

(24) Los motivos fueron muy variados: desde nombres y títulos personales hasta representaciones de individuos, dioses y reyes, breves relatos históricos, normas morales, inscripciones criptográficas y transcripciones de textos (como el Capítulo 30 del Libro de los Muertos, propio de los escarabajos "del corazón"). 

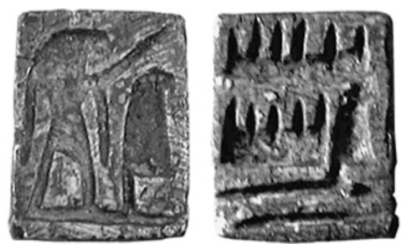

Fig. 6. Plaqueta F0491.

en tamaño $(1,4 \mathrm{~cm} \times 1,1 \mathrm{~cm} \times 0,45 \mathrm{~cm})$. Su forma rectangular presenta ángulos redondeados e inscripciones jeroglíficas en ambas caras. Los signos fueron tallados en bajo relieve y son criptográficos, igual que los del anverso de la plaqueta F0491 arriba considerada (véase Fig. 7).
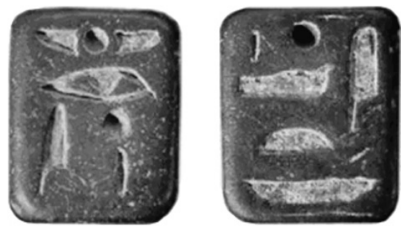

Fig. 7. Plaqueta F0235.

En la inscripción del anverso se registró: $i m n-r^{\complement}$ y en la del reverso: imn $n b \underline{d} t$. El orden de los signos está alterado y para su lectura atribuimos la correspondencia que a continuación indicamos. Los signos del anverso no conservaron sus valores codificados: el sol alado y el ojo (Gardiner 1979: 453) fueron utilizados para transcribir $r^{r}$, en tanto que el criptograma imn fue escrito con el disco solar (Gardiner 1979: 485) sobre una línea vertical, el signo $Z 1$ (Gardiner 1979: 534), y la hoja de junco (Gardiner 1979: 474), pudiendo traducirse la frase completa: "Amón-ra". En el reverso se consignó: imn $n b \underline{d} t$. El primer vocablo, imn, fue escrito con la misma forma criptográfica de escritura empleada en el anverso, en tanto que para $\underline{d} t$ se utilizó la mano (Gardiner 1979: 604) con valor $\underline{d}$ seguida por la terminación femenina $t$ (signo X1, Gardiner 1979: 531), y luego se ubicó la canasta de trabajo (Gardiner 1979: 525). Es probable que esta oración deba traducirse como "Amón, señor de eternidad".

La tercera y última de las plaquetas de Tell el-Ghaba (F0118) fue encontrada en el área II (L1059), en una trinchera exploratoria (Op. 38). Es de esteatita (color 2.5Y 6/3 light yellowish brown) y mide $1,54 \mathrm{~cm} \times 1,55 \mathrm{~cm} \times 0,25-0,35 \mathrm{~cm}$. En sus dos caras tiene diseños decorativos que son de naturaleza simbólica. En el anverso se reconoce un udyat esquemático que fue delineado en bajo relieve y que corresponde al Ojo de Ra. En el reverso se representó un diseño lineal (?), ejecutado con técnica similar y contrapuesto al simbolismo del udyat, que evoca la permanencia (véase Fig. 8).
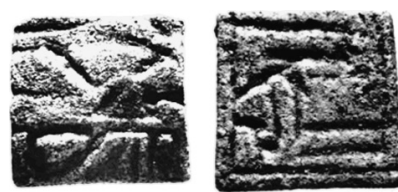

Fig. 8. Plaqueta F0118.

\section{Los escarabajos de Tell el-Ghaba}

El escarabajo fue el amuleto egipcio que por antonomasia simbolizó la renovación y el renacimiento continuo. Considerado como un eficaz protector, alcanzó gran popularidad y difusión, inclusive fuera de las fronteras del antiguo Egipto.

Estaba asociado a la idea de renovación propia del sol - y de autogeneración y era la manifestación del dios Khepry, "el que llega a existir por sí mismo". Como signo jeroglífico (L1 de la lista de Gardiner), el escarabajo hpr representaba los conceptos de transformación dinámica y de conformación.

Fue empleado como amuleto apotropaico, pero también como soporte de textos conmemorativos y, engarzados en anillos o montados como pendientes, su uso como sello personal está bien atestiguado. Los diversos tipos de escarabajos remitían en última instancia a su poder protector, transmitido a través de su impronta en el caso de los sellos. La decoración de la base en particular es indicativa de la específica función cumplida por cada ejemplar y sus dimensiones estuvieron igualmente condicionadas por su uso. ${ }^{25}$

(25) Tres son las formas usadas para escribir en el sistema jeroglífico con valores ocultos: por sustitución de composiciones iconográficas, por perturbación del orden de los signos y por acrofonía (Vandier 1940). Para un listado de los jeroglíficos usados en la composición de los criptogramas véase Jaeger (1982: 294). 
Los escarabajos de Tell el-Ghaba procedentes del área I son ocho: F0015, F0019, F0083, F0622, F0629, F0636A, F0650 y F0655, y dos escaraboides además: F0092 y F0492. Entre ellos pueden distinguirse dos grupos, de acuerdo al tipo de decoración predominante ${ }^{26}$ que poseen: motivos de carácter figurativo e inscripciones jeroglíficas. Tres son los que corresponden al primer grupo: F0015, F0019 y F0083.

El escarabajo identificado como F0015 fue encontrado en superficie y su dorso está mal conservado pero las partes que aún se preservan, la cabeza y clypeus en particular, permiten reconocer una detallada representación naturalista del insecto. Es de esteatita (color 7.5YR 6/4 light brown), de $1,6 \mathrm{~cm} \times 1,15 \mathrm{~cm} \mathrm{x}$ $0,7 \mathrm{~cm}$, y fue conformado y decorado por talla (véase Fig. 9). En la base: se dispusieron cuatro figuras que, de izquierda a derecha y de arriba hacia abajo, pueden reconocerse como representaciones de una mano, un bucranio y los dioses Sobek (cocodrilo) y Bes (enano grotesco). La composición tenía un sentido mágico y se conocen numerosos ejemplos en el Reino Nuevo (Matouk 1966: 39; 249; Petrie 1925: Pl. XV, 066-1067).
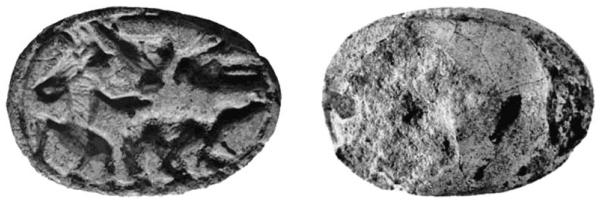

Fig. 9. Escarabajo F0015.

F0019 es un escarabajo que se recuperó durante la excavación del L0018, correspondiente a los escombros del edificio B. Es de faenza (color 5B 7/1 light blueish gray) y sus reducidas dimensiones alcanzan $(\mathrm{en} \mathrm{cm}$ ): 0,95 x 0,7 x 0,5 (véase Fig. 10). Fue moldeado y en la base se registró una composición puramente iconográfica

(26) En algunos casos se combinan signos puramente figurativos con otros de uso codificado en la escritura. No obstante, el carácter ideográfico que en parte tenía la escritura jeroglífica hace que muchas veces la división entre representación figurativa y representación lingüística sea incierta, si no imposible de establecer. de carácter criptográfico, formada por las figuras incisas de una esfinge frente a una jarra-hs, W14 (Gardiner 1979: 529) y el jeroglífico `nh (Gardiner 1979: 508) ubicado horizontalmente por encima de la figura. Con variantes, la composición está documentada en las dinastías 18 y 19 (Newberry 1907: Plate VII). El dorso presenta la forma usual de la representación naturalista tridimensional del escarabajo, con la cabeza, el protórax y la elytra demarcadas por líneas. La primera es aún reconocible a pesar de haberse conservado sólo en parte.
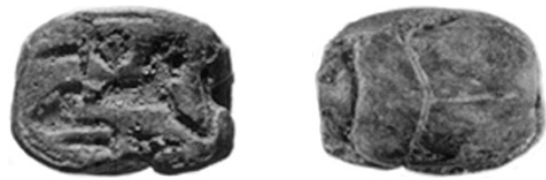

Fig. 10. Escarabajo F0019.

El escarabajo F0083 procede del área I, L0046. Se trata de un ejemplar tallado en esteatita (color: HUE 2,5Y white) que mide $2 \mathrm{~cm} \times 1,35 \mathrm{~cm}$ x 0,7 $\mathrm{cm}$ y fue decorado con bajo relieves (véase Fig. 11). La base presenta la figura incisa de un órix con la cabeza vuelta hacia atrás que está flanqueado por dos cobras - representaciones de la diosa Wadyit. La cabeza, protórax y elytra están delineados en el dorso del escarabajo por talla.

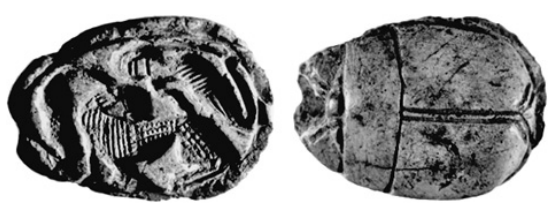

Fig. 11. Escarabajo F0083.

En los tres casos mencionados las composiciones iconográficas de los escarabajos son de carácter simbólico, destacándose la vinculación de algunas de ellas con la realeza.

F0622 es un escarabajo de faenza que procede del L0371 (Fuscaldo 2007: Plate 1, No. 19), uno de los estratos más antiguos del área. ${ }^{27}$

(27) Es una subdivisión del L0266, es decir la arena en la que se excavaron las zanjas de fundación de los edificios A y G. 
Sus dimensiones (en $\mathrm{cm}$ ) son: 1,3 x 0,9 x 0,7 y su color: 2.5.Y 6/3 light yellowish brown (véase Fig. 12).
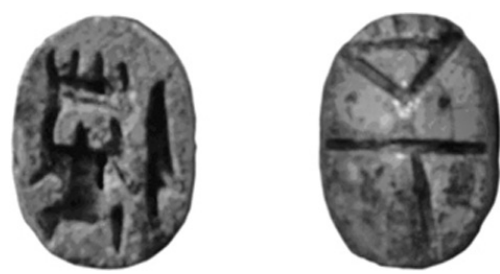

Fig. 12. Escarabajo F0622.

Su decoración está incisa en la base y pintada en el dorso para retratar en forma esquemática el clypeus, la cabeza, el protórax y la elytra del escarabajo. La inscripción jeroglífica de la base es criptográfica y debe leerse: imn-r ${ }^{r}$, "Amón-Ra", correspondiendo la lectura del escarabajo (L1 de la lista de Gardiner) a $r^{\text {c }}$. El trigrama de Amón se representó en este caso por los fonemas $i+m n$, mientras que el nombre del dios solar se expresó en forma alterada por el empleo del signo $h p r(\mathrm{~L} 1)$ por $r^{\mathrm{C}}(\mathrm{N} 5)$.

También el escarabajo inventariado como F0629A, descubierto en L0266 (Fuscaldo 2007: Plate 3 No. 26), es de faenza (color: 10YR 7/2 lignt gray) y su técnica de factura es moldeada. Mide 2,2 cm x 1,8 cm x 1,1 cm, y el dorso presenta una esquemática representación del escarabajo similar a la del anterior y en la base se ubicó una columna de texto (véase Fig. 13). Los jeroglíficos, incisos y pintados, fueron dispuestos en el interior de una cartela flanqueada por dos buitres con las alas desplegadas que representan a Nekhbet. La inscripción reza: $m n-h p r-r^{c}$, "menkheperra" y a ambos lados la duplicación de la diosa enfatiza la realeza del soberano. ${ }^{28}$
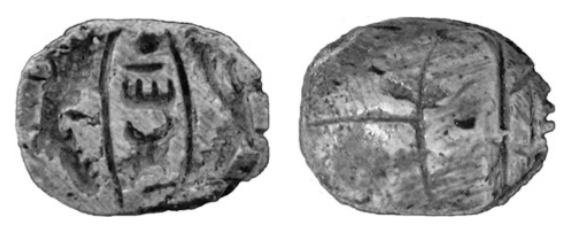

Fig. 13. Escarabajo F0629A.

(28) El diseño de la decoración basal tiene correspondencia simbólica, por ejemplo, con un conjunto de escarabajos de Zagreb (Monnet Saleh, J, 1970: 80-81, n 276-277).
Otro ejemplar de buena factura y bien conservado, es el F0650. Fue descubierto en el L0001 (Fuscaldo 2007: Plate 28, No. 439), que puede ser caracterizado como un nivel de destrucción que alcanza al área II (L1001). Se trata de un escarabajo de faenza (color: 10YR $7 / 2$ lignt gray), de $3,1 \mathrm{~cm} \times 2,3 \mathrm{~cm} \times 1,4 \mathrm{~cm}$, moldeado y en cuyo dorso, de estilo naturalista, se representaron en detalle la cabeza, el protórax y la elytra del insecto (véase Fig. 14). La inscripción jeroglífica basal, incisa y pintada (chart 1 for glay $7 / 15 \mathrm{GY}$ light greenish gray), dice: $d i$ b3stt(?) 'nh nfr, "Bastet da una vida buena". ${ }^{29}$
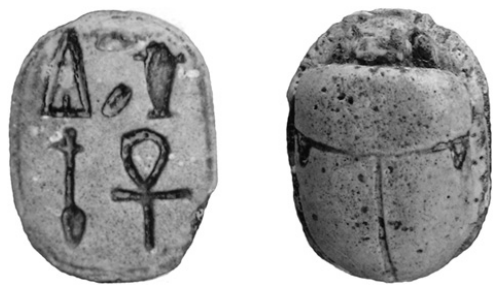

Fig. 14. Escarabajo F0650.

No contamos con los registros fotográficos de los escarabajos F0636A y F0655, y su documentación textual y gráfica sólo permite describirlos parcialmente aquí.

F0636A fue recogido en el L0326, un fogón posterior al edificio A. Es un ejemplar de faenza moldeada que está completo pero con muy mala preservación. ${ }^{30}$ Sus dimensiones son $2,3 \mathrm{~cm} \times 1,8 \mathrm{~cm} \times 1,1 \mathrm{~cm}$. Las líneas que decoraban el dorso están desgastadas y la base conserva figuras que no podemos identificar a partir del esquemático dibujo con que fue documentado gráficamente. El dorso sólo se conserva en parte (véase Fig. 15).

En cuanto al escarabajo F0655, ${ }^{31}$ también procede de L0001 (Fuscaldo 2007: Fig. 40: 37, No 440), que posiblemente representa el final de la ocupación saíta. Es un ejemplar de faenza (color 10YR 7/2 light gray), moldeado, de 0,9 $\mathrm{cm} \times 1,2 \mathrm{~cm} \times 0,6 \mathrm{~cm}$, cuya decoración basal

(29) Para otras variantes de la composición véase Petrie (1925: Pl. XII, 722-724).

(30) Dibujo n $936 / 2002$.

(31) Dibujo ${ }^{\circ} 443 / 1999$. 

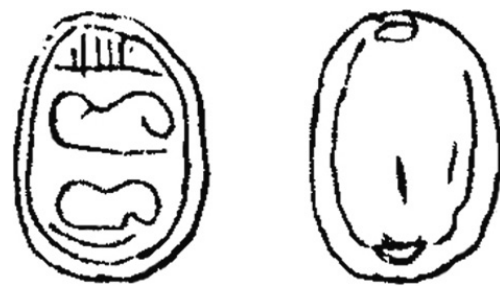

Fig. 15. Escarabajo F0636A.

está en mal estado de conservación. Su dorso es de carácter naturalista y fue representado con líneas incisas (véase Fig. 16).
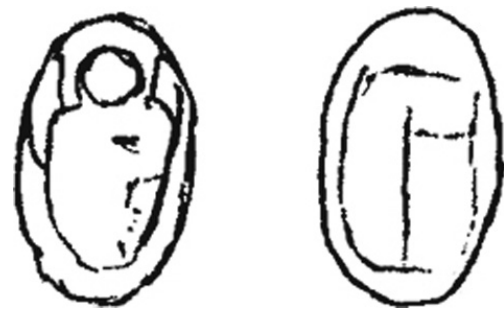

Fig. 16. Escarabajo F0655.

Finalmente, del área I proceden un escaraboide en cuyo dorso fue tallada la figura de la diosa hipopótamo (F0092) y otro (F0492) cuya decoración dorsal presenta un diseño lineal para el que no encontramos paralelos.

El ejemplar F0092 es de esteatita (color: 7.5YR 6/4 light brown), de $2,25 \mathrm{~cm} \mathrm{x} 1,7 \mathrm{~cm} \mathrm{x}$ 0.65 y su decoración se esculpió en bajo relieve. El estrato de destrucción e incendio en que se encontraba (L0056) corresponde a la porción inferior del L0001.

La figura de Tueris que decora el dorso muestra a la diosa hipopótamo erecta sobre sus patas traseras y sosteniendo con una de las delanteras un ramo y con la otra el signo jeroglífico de la protección, $s^{32}$ (véase Fig. 17). En cuanto a la base: una inscripción jeroglífica de carácter criptográfico ocupa la superficie. El texto ofrece una multiplicación del trigrama de Amón. ${ }^{33}$ La primera versión del nombre del

(32) Similar a uno de los escaraboides publicado por Matouk (1966: fig. 144).

(33) Para las diversas formas de escribir criptográficamente el nombre de Amón - o Amón-Ra -, véase Matouk (1966: 64 , No $1304-1306$ y 1308). Para variantes próximas a las dios es imn-r r, escrita con el signo L2, la abeja (Gardiner 1979: 477) por $i$ y la cartela V10 (Gardiner 1979: 522) por el bilitero $m n$, y el vocablo $r^{\complement}(\mathrm{N} 5)$ con su valor y posición jerárquica de precedencia usuales. La segunda versión se dispuso dentro de la cartela y sigue el patrón de los escarabajos "menkheperra", transcribiendo el trigrama de Amón con los signos Y5 (Gardiner 1979: 534) por $i$, L1 (Gardiner 1979: 477) por $m n$ (con determinativo fonético $n$ ) y $r^{c}$ en su posición de precedencia y con su valor usual. A la izquierda se desarrolló la tercera versión del nombre divino, escrito con la pluma de Maat
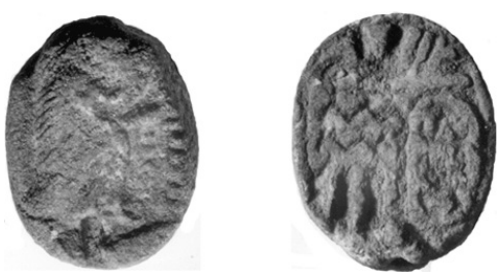

Fig. 17. Escaraboide F0092.

en sustitución de $i$ (L1), la onda de agua (N35) por $m n(\mathrm{Y} 5)$ y la hoja de caña (M17) por $n(\mathrm{~N} 35)$.

El segundo escaraboide (F0492) se encontró completo en el L0001. La buena calidad de su factura es aún perceptible a pesar del deterioro sufrido a causa de la fragilidad del material (faenza) con que se manufacturó y del sedimento que lo contenía, identificado como un nivel de destrucción. De color 2.5Y 4/1 light gray $2.5 \mathrm{Y} 5 / 1$ grayish brown, sus dimensiones son $1,5 \mathrm{~cm} \times 1,9 \mathrm{~cm} \times 0,5 \mathrm{~cm}$. Fue modelado sobre el patrón oval más característico de los amuletos sellos con forma de escarabajo y la decoración dorsal se realizó por incisiones con líneas de orientación longitudinal (véase Fig. 18). No se preservó la inscripción de la base y a partir de su registro gráfico ${ }^{34}$ no fue posible encontrar paralelos bien definidos.

Los escarabajos del área II son cuatro, inventariados como F0166, F0274, F0434 y F640. Dos de ellos (F0274 y F0434) corresponden al

registradas en la base de F0092 véanse Petrie (1925: 1263 . 1267) y Jaeger (1982: 286, ills. 670 y 672).

(34) Dibujo n² 271/1998. 


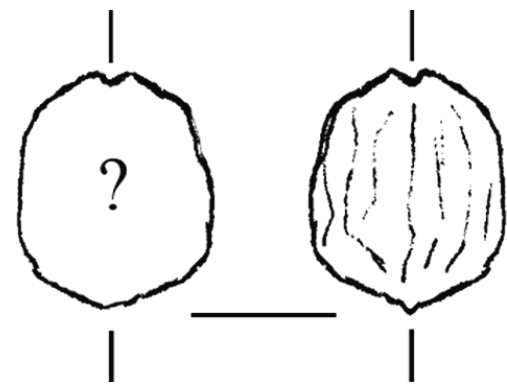

Fig. 18. Escaraboide F0492.

tipo "menkheperra" arriba mencionado, en tanto que los otros dos (F0166 y F0640A) presentan una decoración basal simbólicamente diferente y en la que se combinaron elementos figurativos y signos jeroglíficos.

El escarabajo F0274 es de faenza moldeada (color: 10 YR $8 / 4$ very pale brown) y de tamaño pequeño: $0.78 \mathrm{~cm} \times 0.65 \mathrm{~cm} \times 0.4 \mathrm{~cm}$ (véase Fig. 19). Procede del L1091 (Fuscaldo 2007: Plate 7, No. 1), un piso bien definido descubierto en el curso de una operación exploratoria realizada por debajo del edificio C. La decoración del dorso tiene un delineado inciso que representa las formas naturales del escarabajo, mientras que la inscripción jeroglífica $m n-h p r-r^{c}$, "menkheperra" fue grabada en el interior del óvalo que enmarca la base del escarabajo. A diferencia de otros ejemplares de su tipo, el jeroglífico hpr (el escarabajo), se encuentra orientado horizontalmente, como se observa en algunos escarabajos de la dinastía 18 (Matouk 1966: $59, n^{\circ} 564,566$ y 569; 60, n 570-580 y 582-583).
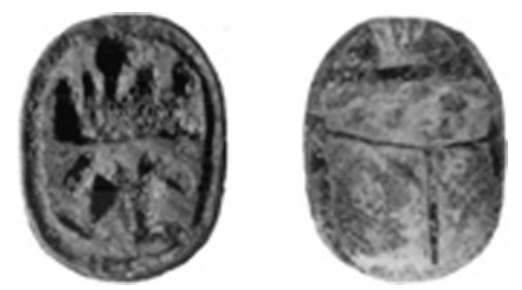

Fig. 19. Escarabajo F0274.

El segundo escarabajo "menkheperra" del área II, F0434 fue hallado en superficie (BG/ 70-BG/71). Es de faenza moldeada (color 5GY $7 / 1$ light greenish gray) y sus medidas son 2,8 $\mathrm{cm} \times 2,1 \mathrm{~cm} \times 1,2 \mathrm{~cm}$ (véase Fig. 20). En el dorso lleva incisa una inscripción jeroglífica que está dispuesta en columna en el interior de una cartela, coronada por una doble pluma, que reza: $m n-h p r-r$ ', "menkheperra". ${ }^{35} \mathrm{La}$ decoración basal presenta la figura de un león en marcha, ${ }^{36}$ representación icónica del faraón que refuerza la composición por su referencia al soberano como Menkheperra y por la doble pluma asociada a Maat, el orden cósmico que el gobernante debía mantener.

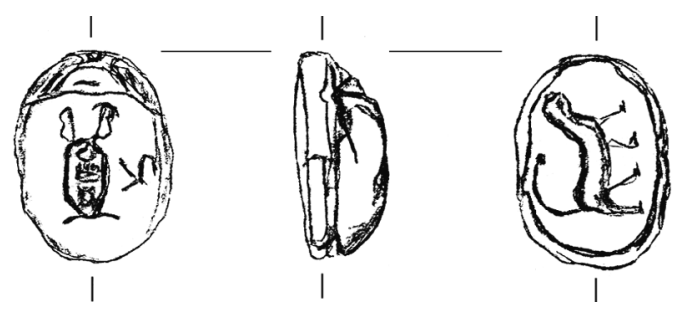

Fig. 20. Escarabajo F434.

El escarabajo F0166 procede del L1004 y fue descubierto durante la excavación de una trinchera exploratoria (trinchera Norte A); sería algo posterior al L0001 (Fuscaldo 2007: Plate 8, No. 142). Fue tallado en esteatita (color: HUE 10YR $8 / 2$ very pale brown), sus medidas (en $\mathrm{cm}$ ) son: $5 \times 1,3 \times 0,7$, y su dorso requebrajado le da el aspecto de un armadillo (véase Fig. 21). No obstante, la detallada configuración de la cabeza y parte delantera permite reconocer una representación naturalista del dorso del escarabajo.
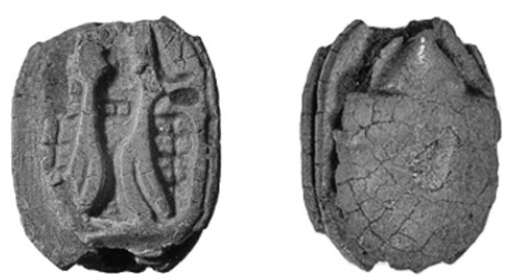

Fig. 21. Escarabajo F0166.

(35) A partir de su descripción y registro gráfico (dibujo $\mathrm{n}^{\circ}$ 266/1998). El dorso es similar al publicado por Petrie (1925: Pl. XXVII, 28).

(36) Similar al ejemplar publicado por Petrie (1925: Pl. XVIII, 1395). En el dibujo no se documentó el disco solar, que en la publicación de Petrie se ubicó por encima de la figura del león. 
La base fue decorada con una inscripción incisa compuesta por una jarra-hs (W14) sobre la que se lee el signo jeroglífico $r^{\complement}$ y que está flanqueada por dos figuras de la diosa Wadyit con la corona roja. ${ }^{37}$ La lectura $h s(y)-r^{c}$ del texto jeroglífico puede traducirse como "el favorito de Ra”, en tanto que la presencia duplicada de la figura de la diosa remite a la realeza del Bajo Egipto que la divinidad protege. Así, el texto puede interpretarse como epíteto del soberano cuya realeza del Bajo Egipto las diosas sustentan.

Por último, cabe mencionar al escarabajo inventariado como F0640A (Fuscaldo 2007: Fig. 7: 3 No 27), del que tampoco poseemos documentación fotográfica. ${ }^{38}$ Se lo recuperó durante la realización de la Operación 99/6 en el L1040, un sedimento laminar subyacente al edificio L. Fue moldeado en faenza (color: 10YR $7 / 2$ light gray) y sus dimensiones son $0,9 \mathrm{~cm} \times 1,2$ $\mathrm{cm} \times 0,7 \mathrm{~cm}$. Se conserva completo - aunque fragmentado en dos partes $-\mathrm{y}$ fue decorado con líneas incisas que en el dorso representaron la parte superior del escarabajo y en la base un esquemático motivo de voluta simple con simetría especular en sentido longitudinal (véase Fig. 22).
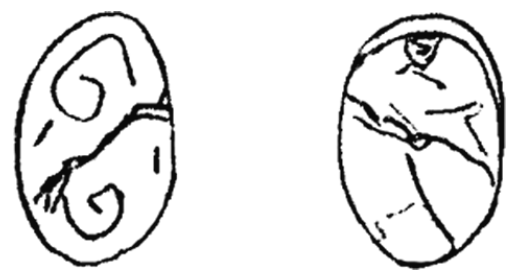

Fig. 22. Escarabajo F640A.

\section{Consideraciones finales}

El conjunto de sellos amuletos que caracteriza los materiales epigráficos de Tell el-Ghaba muestra una diversidad relativa en su tipología y factura, a la vez que comparten los rasgos propios de su carácter protector.

De las 19 piezas analizadas, 14 proceden del área I y representan un total próximo al 74

(37) Sólo preservada en la figura de la derecha.

(38) Dibujo no 453/1999.
$\%$ de los materiales con epigrafía del sitio, registrándose para el área II sólo 5 , lo que corresponde a cerca del 26\% de los hallazgos.

El hecho que hubiera una mayor presencia de este tipo de hallazgos en el área I podría señalar su empleo por los funcionarios que ocuparon esa área residencial. Por otra parte, el área I era además el sector industrial del sitio, en donde se encontraron hornos con canalículos que conservaban escoria de faenza. Esa circunstancia podría ser indicativa de una producción local de amuletos (Crivelli 2006: 21), parte de los cuales fueron descubiertos durante las excavaciones de la Misión Argentina en el sitio. No obstante, tanto la diversidad absoluta de las piezas como la ausencia de ejemplares pertenecientes a una misma serie resultan contrarias a esto.

Las diferentes opciones de materiales y los requerimientos técnicos para la factura de cada uno de los amuletos son indicativos de su valor simbólico y del grupo social al que su producción estaba destinada, probablemente identificable con la elite local.

Por otra parte, el hecho de llevar muchos de esos amuletos una decoración estrechamente vinculada a la iconografía y/o la fraseología real o vinculada al dios del imperio, Amón, sugiere una pertenencia de sus destinatarios al entorno estatal.

Respecto de la naturaleza de los amuletos analizados, es interesante señalar la frecuencia relativa de las inscripciones criptográficas, que enmascaran el nombre del dios Amón con inversiones de la posición de los signos y empleo con valores diferentes de los codificados en el sistema jeroglífico de escritura. La proporción de las composiciones predominantemente iconográficas, lingüísticas o criptográficas de los textos es, excepto dos casos que no pueden ser definidos, bastante equilibrada: seis son de carácter iconográfico, cinco lingüístico y cinco criptográfico.

Por último, el estudio comparativo de los amuletos con epigrafía de Tell el-Ghaba permite establecer algunos paralelos con los de otros sitios arqueológicos, lo que contribuye a la interpretación del sitio y su cronología. En particular las composiciones figurativas 


\begin{tabular}{|c|c|c|c|c|}
\hline \multicolumn{5}{|c|}{ Material epigráfico del área I } \\
\hline Inv. No & Loci & Objeto & Decoración & Texto \\
\hline F0015/95 & superf. & escarabajo & Bes + mano + bucranio + Sobek & iconográfico \\
\hline F0019/95 & L0018 & escarabajo & esfinge $+h s+c_{n h}$ & iconográfico \\
\hline F0072/95 & L0008 & amuleto-sello & Heket/inscripción ilegible & lingüístico \\
\hline F0083/95 & L0046 & escarabajo & león flanqueado por 2 cobras $+r^{c}$ & iconográfico \\
\hline F0092/95 & L0056 & escaraboide & Tueris $/ i m n-r^{c}+m n-h p r-r^{c}($ en cartela $)+i m n$ & criptográfico \\
\hline F0235/96 & L0211 & plaqueta & $i m n-r^{c} / i m n n b \underline{d} t$ & criptográfico \\
\hline F0491/98 & L0001 & plaqueta & $\mathrm{imn} / \mathrm{mn} \mathrm{imn}$ & criptográfico \\
\hline F0492/98 & L0001 & escaraboide & no preservada & — \\
\hline F0622/99 & L0371 & escarabajo & $i m n-r^{c}$ & criptográfico \\
\hline F0629/99 & L0266 & escarabajo & $m n-h p r-r^{\complement}$ (en cartela) flanqueada por 2 halcones & lingüístico \\
\hline F0636A/02 & L0326 & escarabajo & figuras no identificadaa & iconográfico \\
\hline F0650/99 & L0001 & escarabajo & dit $p^{\complement} t h r n f r{ }^{\complement} n h$ & lingüístico \\
\hline F0655/99 & L0001 & escarabajo & no preservada & \\
\hline F1000/01 & L0271 & udyat & $m n h p r-r^{r}$ & lingüístico \\
\hline
\end{tabular}

\begin{tabular}{|c|c|c|c|c|}
\hline \multicolumn{5}{|c|}{ Material epigráfico del área II } \\
\hline Inv. No & Loci & Objeto & Decoración & Texto \\
\hline F0118/ & L1059 & plaqueta & udyat / diseño lineal $?$ ? & iconográfico \\
\hline F0166/96 & L1004 & escarabajo & $h s(y)-r^{c}+2$ cobras & lingüístico \\
\hline F0274/97 & L1091 & escarabajo & $m n-h p r-r^{r}$ & criptográfico \\
\hline F0434A/98 & superf. & escarabajo & $\begin{array}{l}\text { león en marcha }+m n-h p r-r^{c} \\
\text { (en cartela flanqueada por } 2 \text { plumas) }\end{array}$ & criptográfico \\
\hline F0640A/96 & L1040 & escarabajo & Volutas & iconográfico \\
\hline
\end{tabular}

estandarizadas ${ }^{39}$ y los escarabajos del tipo "menkheperra" son de utilidad para corroborar en parte la cronología establecida en primer término en base a la datación cerámica. En líneas generales se verifica una aceptable correspondencia entre los períodos en los que se pueden datar los amuletos con epigrafía de Tell el-Ghaba y los fechados propuestos para las áreas investigadas.

(39) Como la del escarabajo F0083, similar a la plaqueta CGC 12.667 (Reisner 1958: Pl. IV), de faenza moldeada y que presenta como variante una representación de Bes en una de sus caras y la mano y el cocodrilo en la otra. Los escarabajos decorados con la esfinge, están bien atestiguados en las dinastías 18 y 19, y los signos de un ejemplar de la 19 (Newberry 1907: 160; Pl. VII, 36642) coinciden exactamente con la distribución de los ubicados en la base de F0019. 
PEREYRA, M.V. The epigraphic material of Tell el-Ghaba (Northern Sinai, Egypt). Revista do Museu de Arqueologia e Etnologia, São Paulo, 19: 257-271, 2009.

Abstract: The purpose of this paper is to interpret the epigraphic material unearthed in Tell el-Ghaba during the excavations carried out between 1995 and 2000 by the Argentine Archaeological Mission. After the cataloging of finds, the translation, and the interpretation of inscriptions - as well as the decoding of iconography -, the decorative motifs were compared with similar ones held on objects with epigraphy coming from contemporary sites, and finally they were studied on bibliographical bases. As a result, we can observe that: 1 - the nature of the analyzed amulets is cryptographic in many cases; 2 the texts registered on the amulets are mainly linked to royalty and Amun theology; 3- the majority of finds belongs to area I, industrial and residential in character.

Keywords: Tell el-Ghaba - Epigraphy - Amulet - Scarab - Plaque - Horus eye - Cryptography.

\section{Referencias bibliográficas}

DRIOTON, É.

1940 Recueil de cryptographie monumentale. Annales du Service des Antiquités de l'Égypte 40: 305-427.

FUSCALDO, P. (Ed.)

2007 Tell el-Ghaba I. A Saite Settlement in North Sinai Egypt (Argentine Archaeological Mission, 1995-2004), I. The Catalogue. Colección Estudios, 5. Buenos Aires, CONICET.

CRIVELLI MONTERO, E.

2006 El extremo oriental del Delta en el pasado y en la actualidad. In: Basílico, S.; Lupo, S. (Eds.) Tell El-Ghaba, norte de Sinai, Egipto. Alimentación, producción e intercambio. Buenos Aires, Dunken: 11-31.

CRIVELLI MONTERO, E.; COHEN, C.; CHAUVIN, A.

2002 Tell el-Ghaba, Sinai Norte, Egipto: el asentamiento y la economía. In: Actas del $1{ }^{\circ}$ Congreso Nacional de Arqueología Histórica. Buenos Aires, Corregidor: 833 841.

JAEGER, B.

1982 Essai de classification et datation des scarabées Menkhéperrê. Fribourg. Orbis Biblicus et Orientalis, Serie archaeologica 2.
MATOUK, F.S.

1966 Corpus du scarabée égypien, 2. Analyse thématique. Beyrouth, Académie Libanaise.

MÜLLER H.W.

1954 Skarabën und andere Siegel Perlen mit Inschriften und Ägyptische Siegelzylinder. In: Müller, H.W. Skarabäen-Sammlung des Frei Herrn F.W. von Bissing. Kunst-Auktion 20, Dritter Teil. Stutgarter, Kunst Kabinett R.N. Ketterer: 44-60.

MONNET SALEH, J.

1970 Les antiquités égyptiennes de Zagreb. Catalogue raisonné des antiquités égypienes conservées au Musée Archéologique de Zagreb en Yougoslavie. Paris; La Haye: Mouton.

NEWBERRY, P.

1907 Scarab-shaped Seals. Catalogue General des Antiquités égyptiennes du Musée du Caire. Le Caire, Institut Français d'Archéologie Orientale.

PEREYRA, M.V.; ZINGARELLI, A.P.

2002 Tell el-Ghaba. Un asentamiento en la antigua frontera sinaítica de Egipto. In: Actas del $1^{\circ}$ Congreso Nacional de Arqueología Histórica. Buenos Aires, Corregidor: 853-863. 
PEREYRA, V.; ZINGARELLI, A.

2005 The ancient Sinaitic frontier of Egypt. In: Fuscaldo, P.; Crivelli, E.V.;

Zingarelli, A. The Preliminary Report of the Three Campaigns of the Argentine Archaeological Mission at Tell El-Ghaba, North Sinai, Egypt. 1995-1997 (Excavation and Study Seasons). Revista de Estudios de Egiptología, 6/7: 9-15.

PETRIE, $\mathrm{F}$ et alii.

1925 Buttons and Design Scarabs. Illustrated by the Egyptian Collection in University College. London, Bernard Quaritch.
REISNER, G.A.

1958 Catalogue Général des Antiquités Égyptiennes du Musée du Caire. Amulets, 2. Le Caire, Institut Français d'Archéologie Orientale.

ROSSO, A.M.; FANTECHI, S.; ARBOLAVE, G.

2002 Tell El-Ghaba: Los hallazgos especiales: los materiales y su conservación. Significación histórica. In: Actas del $1^{\circ}$ Congreso Nacional de Arqueología Histórica. Buenos Aires, Corregidor: 875-886.

ZINGARELLI, A.

2005 Acerca de la frontera oriental del Egipto Antiguo. Revista de Estudios de Egiptología, 6/7: 171-184. 